

\title{
L'aménagement des parkour-parks : les espaces dédiés pour les activités ludo-sportives comme outils d'inclusion? Études de cas à Nantes et à Rennes
}

Robin Lesné, Christophe Gibout, Florian Lebreton

\section{- To cite this version:}

Robin Lesné, Christophe Gibout, Florian Lebreton. L'aménagement des parkour-parks: les espaces dédiés pour les activités ludo-sportives comme outils d'inclusion? Études de cas à Nantes et à Rennes. Loisir et Société / Society and Leisure, 2019, 42 (3), pp.378-400. 10.1080/07053436.2019.1681799 . hal-02383209

\section{HAL Id: hal-02383209 \\ https://hal.science/hal-02383209}

Submitted on 1 Apr 2021

HAL is a multi-disciplinary open access archive for the deposit and dissemination of scientific research documents, whether they are published or not. The documents may come from teaching and research institutions in France or abroad, or from public or private research centers.
L'archive ouverte pluridisciplinaire HAL, est destinée au dépôt et à la diffusion de documents scientifiques de niveau recherche, publiés ou non, émanant des établissements d'enseignement et de recherche français ou étrangers, des laboratoires publics ou privés. 


\title{
L'aménagement des parkour-parks : les espaces dédiés pour les activités ludo-sportives comme outils d'inclusion ? Etudes de cas à Nantes et à Rennes
}

\author{
Robin Lesné*, Christophe Gibout et Florian Lebreton \\ Univ. Littoral Côte d'Opale, Univ. Lille, EA 4477, TVES - Territoires Villes Environnement \& \\ Société, F-59140 Dunkerque, France
}

Skating sports have dominated the field of studies in human and social sciences on sportsleisure practices for a long time, particularly because of their visibility in cities by the establishment of open spaces: skate parks. However, since the 1990s, these activities that invest urban spaces are diversifying. Parkour is one of them and presents the originality of questioning the (skate and/or parkour) parks as it is an urban planning tool because he also experiences, henceforth, the development of open and public facilities dedicated to this activity. Based on a literature review and a study conducted in Rennes and Nantes, we question this fact of the park and show that, beyond its urban planning issues, it constitutes (1) a lever of the emergence of renewed youth citizenship; and (2) a vehicle for building a not only sporty, but more broadly recreational, urbanity.

Keywords: skateboarding, parkour, sport open space, recreational urbanity, youth citizenship

Les sports de glisse ont longtemps dominé le champ des études en sciences humaines et sociales sur les pratiques ludo-sportives, notamment du fait de leur visibilité dans les villes par l'aménagement d'espaces dédiés : les skate-parks. Pourtant, depuis les années 1990, ces activités qui investissent les espaces urbains connaissent une diversification. Le parkour fait partie de celles-ci et présente l'originalité d'interroger les (skate et/ou parkour) parks comme outil d'aménagement en ce qu'il connait lui aussi, désormais, le développement d'équipements ouverts et publics qui lui sont dédiés. A partir d'une revue de littérature et d'une étude menée à Rennes et à Nantes, nous interrogeons cette réalité du park et démontrons qu'au-delà de ses enjeux aménagistes, il constitue (1) un levier de l'émergence d'une citoyenneté juvénile renouvelée et (2) un vecteur de la construction d'une urbanité, non seulement sportive, mais plus largement récréative.

Mots clés : skate, parkour, espace sportif dédié, urbanité récréative, citoyenneté juvénile

*Corresponding author. Email: robin.lesne@etu.univ-littoral.fr 


\section{Du rider au traceur, glissement de la réflexion}

Les sports de glisse en milieu urbain sont mobilisés en science de manière plurielle. Cette diversité s'observe dans les disciplines de rattachement des chercheurs, dans les approches dépassant l'entrée sportive et/ou des loisirs, mais aussi dans les supports de publication dont les thématiques éditoriales semblent a priori éloignées. Les skate-parks illustrent cette réalité : ils sont un objet étudié en architecture, aménagement et urbanisme (Nemeth, 2006; Shannon et Werner, 2008 ; Chiu, 2009; Taylor et Khan, 2011), en sciences politiques (Michenaud, 2016 ; Turner, 2017) ou encore en sociologie (L'Aoustet et Griffet, 2001 ; Vieille Marchiset, 2003 ; Savari, Chaves et Machemehl, 2011 ; Gibout et Lebreton, 2014 ; Riffaud, 2017). Dans un objectif de renouvellement de la réflexion et des approches scientifiques, nous proposons d'interroger les espaces dédiés au regard de l'émergence plus contemporaine des parkour-parks (Figure 1). Compléter les études sur la glisse urbaine par le parkour ambitionne d'enrichir la compréhension de cet outil, de ses enjeux et de ses perspectives.
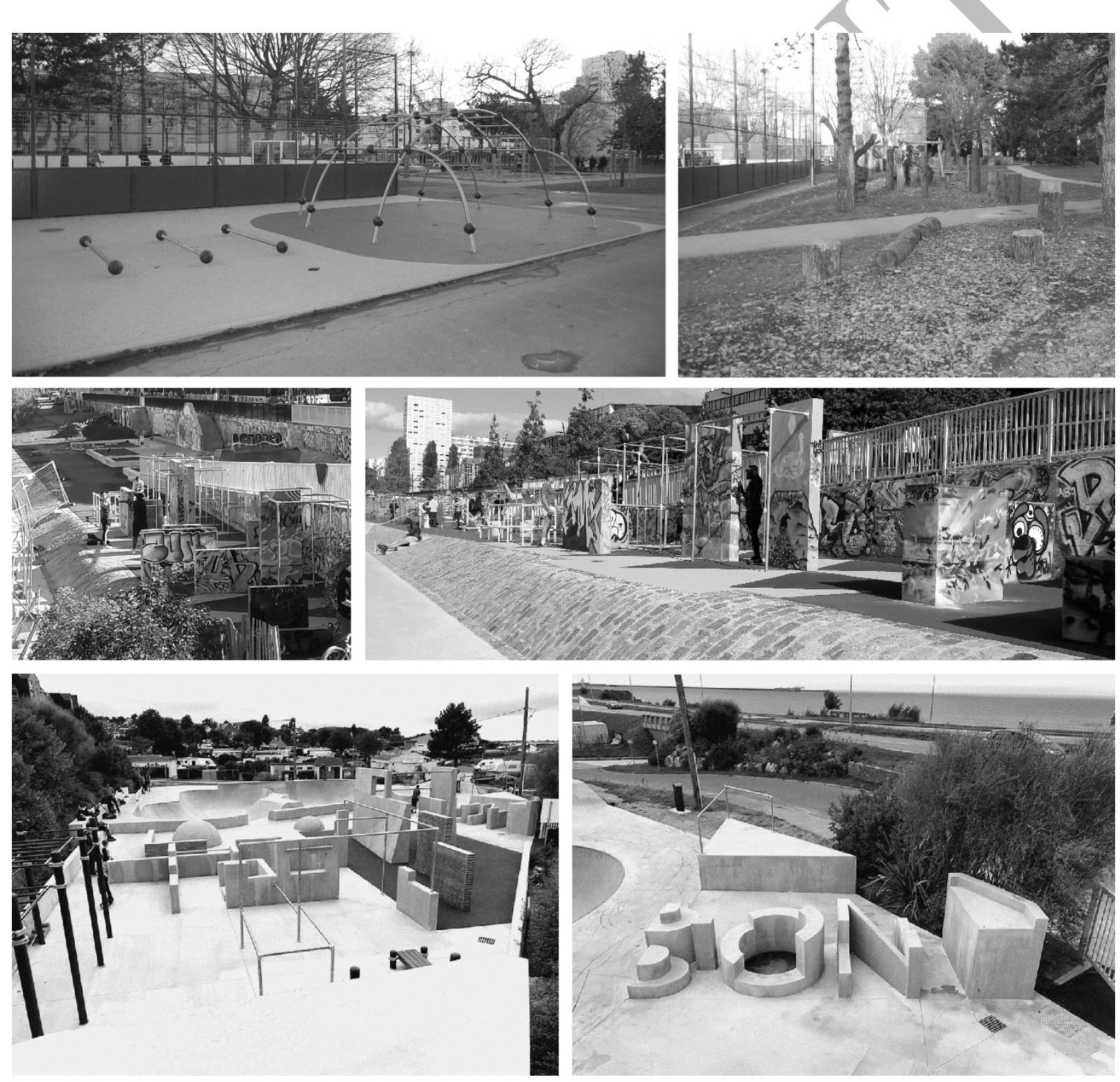

Figure 1. De haut en bas, exemples d'espace dédié à Rennes et de parkour-parks à Nantes et à Cherbourg (Robin Lesné et https://www.facebook.com/TeamB.0N/, 2018) 
Le parkour "est un art du déplacement et une activité physique dont la visée est un déplacement libre et efficace en dehors des voies de passage préétablies et se confrontant à tous types d'environnements et d'éléments » (Gibout et Lebreton, 2014, p. 71) impliquant le détournement d'usage (Figure 2). Il revêt des caractères informel et transgressif à repositionner dans le contexte récent d'institutionnalisation : montages associatifs (Art du déplacement academy et Art in Motion pour Nantes, Ouest Parkour à Rennes) ; développement d'espaces dédiés pilotés par les pouvoirs publics. Le parkour est une activité à la fois physique et ludique, mettant en mouvement le corps athlétique et investissant l'espace par le jeu, comme peuvent l'être les sports de glisse en skate-park et dans la rue.

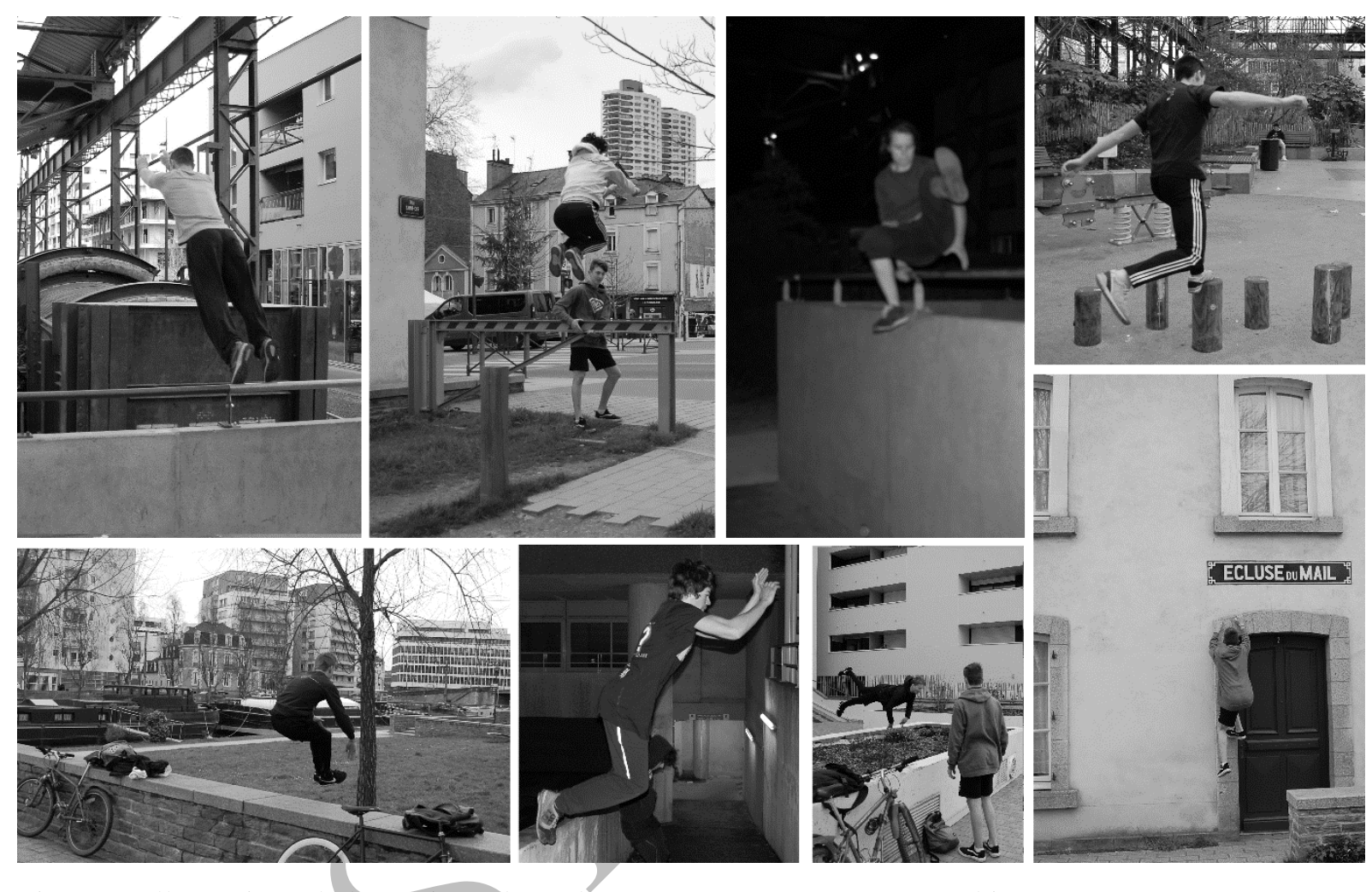

Figure 2. Illustrations de la pratique du parkour à Rennes et à Nantes (Robin Lesné, 2018).

Croiser l'étude du parkour et des sports de glisse vise à mettre au jour la réflexion sur les urbanités sportives contemporaines par l'émergence des espaces dédiés, d'une part, et récréatives dans leur forme street, d'autre part. Relevant des sports urbains et des ludo-sports du fait de l'importante porosité de leur frontière, il convient de revenir sur ces concepts pour mieux appréhender le positionnement de notre démarche. Augustin (1997) introduit le terme de ludo-sport face à l'émergence d'activités physiques présentant une recherche récréative par une forme plus « aventurière » à travers la conquête des grands espaces de nature et la reconquête des espaces urbains. Il complète ce concept en insistant sur le caractère organisationnel autorégulé par émancipation du système fédéral de performance et de compétition, sur l'éloignement du modèle du club sportif et sur le développement de sociabilités informelles hors des institutions (Augustin, 2002). Les ludo-sports s'inscrivent donc principalement dans une dynamique émancipatrice. Pour ce qui est des sports urbains, le concept se distingue notamment par une dimension plus politique. Lors des premiers Etats généraux du sport urbain, en 2009 à Paris, la Secrétaire d'État chargée des sports déclarait: "Depuis des années, les sports urbains ont connu une forte croissance. Mais cette croissance s'est faite, c'est là le problème à l'écart du monde sportif traditionnel et sans aucune reconnaissance des pouvoirs publics.»( $\mathrm{R}$. Yade, 16/12/2009). Ce discours illustre la mobilisation des terminologies de «sports urbains » et «sports de rue » dans le champ politique, la problématisation de leurs appréhension et distance institutionnelles et leur logique contre-culturelle 
« rebelle» (Augustin, 2001). La catégorisation scientifique relative aux sports urbains provient de la première étude menée sur les pratiques physiques des jeunes en ville comme «sports de la cité »par opposition à un «sport dans la cité » (Chantelat, Fodimbi et Camy, 1996). La ville n'est pas un simple lieu de présence mais elle est un contexte socio-spatiale urbain, un espace de création culturelle, i.e. une forme d'urbanité qui est le terreau propice à l'invention sociale et l'émergence de nouvelles pratiques sportives. Ce que Lebreton (2009) appelle d'ailleurs l'urbanité ludique, par opposition à l'urbanité sportive. Les effets de la concentration de l'habitat, de la verticalité, de la présence de délaissés temporaires, d'habitants et d'usagers de l'espace favorisent des « chemins de traverse » et des « usages détournés » (de Certeau, 1990) de l'espace. Par exemple, Travert (1999) a pu montrer un effet de territoire urbain sur la pratique du « football de pieds d'immeuble ». Gibout et Mauny (2009) ont pu mettre en exergue, via le paradigme du « sport sauvage ", l'existence d'un effet différentiant de territoire et de culture. La distinction entre ludo-sports et sports urbains se fait sur leurs divergences des sports traditionnels (Figure 3). Le parkour et les sports de glisse urbaine sont des ludo-sports en ce qu'ils présentent des caractéristiques de fonctionnement émancipatrices et ils sont des sports urbains car ils ne peuvent émerger que de la ville dans un rapport aux normes sociales oppositionnel, voire conflictuel. Les pratiquants du parkour revendiquent, comme certains skateurs, une distance culturelle avec le modèle sportif traditionnel rejeté à l'origine et encore aujourd'hui par certains " puristes » (Bornaz, 2013), traceurs de premières générations (Lebreton, 2017). "Traceurs» est le nom donné aux pratiquants, jeu de mot entre le fait qu'ils tracent leur propre chemin dans la ville et le principe de ne laisser aucune trace du passage. La comparaison entre le parkour et les sports de glisse urbaine n'est pas envisageable au motif de leurs nombreuses différences, notamment l'utilisation d'un outil ou du seul corps. L'objectif ici est de mobiliser l'évolution et la diversification des ludo-sports pour interroger les parks, de glisse urbaine et de parkour, comme outil d'inclusion. Plus précisément, au-delà de l'analyse réunissant ces pratiques dans des catégories d'activités, le développement de leurs parks devient un élément de leur convergence autour du renouvellement de la citoyenneté des pratiquants et de la société.

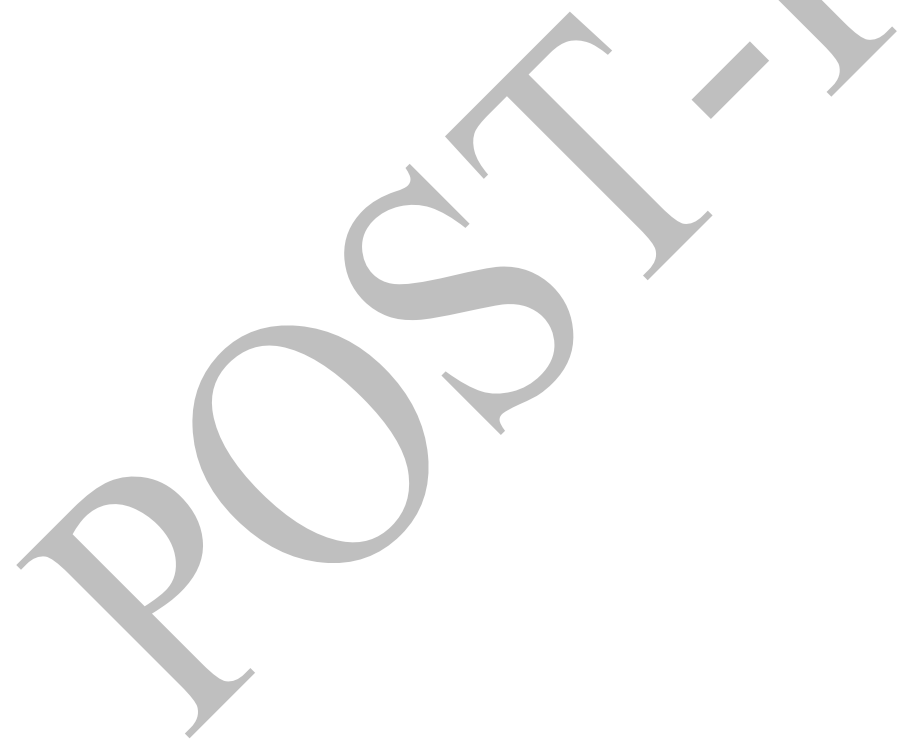




\section{LUDO- SPORTS SPORTS SPORTS TRADITIONNELS URBAINS}



Figure 3. Sports urbains et ludo-sports face au mouvement sportif traditionnel

L'étude repose sur un travail universitaire de Master dans le cadre du lancement d'une recherche doctorale. Il a permis la construction d'un projet de thèse lancé en novembre 2018 s'intéressant à la ville récréative à travers l'étude du parkour et de l'urbex. Les principaux résultats empiriques proviennent d'une enquête de terrain menée à Rennes et à Nantes entre février et mars 2018. La réflexion s'intéressait à l'institutionnalisation du parkour par l'aménagement des parkour-parks. Dix observations interactives, i.e. des phases d'observation au positionnement ouvert du chercheur en échange avec les pratiquants, ont été réalisées (Table 1). Elles ont permis la prise de contact avec les traceurs, la construction d'une base de données sociodémographiques, la récolte d'un verbatim à l'appréciation du chercheur et l'analyse technique de la pratique afin de comparer ses formes street et en park. De plus, 17 entretiens compréhensifs semi-dirigés ont été menés (Table 2) auprès de 19 personnes : pratiquants $(\mathrm{P})$, responsables associatifs (AP), acteurs des services municipaux (T), professionnels de l'aménagement (PA) et un élu (E). Ils étaient séquencés autour de quatre sujets : le parcours de la personne, la pratique du parkour, les traceurs et les parkour-parks. Ils n'ont pas été enregistrés aussi les citations sont des propos jugés pertinents et retranscris en direct. L'analyse a consisté en la synthétisation des discours des interviewés au regard d'une grille de thèmes. Parmi les pratiquants, quatre ont été interrogés lors d'entretiens en binôme.

Table 1. Récapitulatif des phases d'observation 


\begin{tabular}{lccccc}
\hline Code & Temporalité & $\begin{array}{c}\text { Durée } \\
\text { (minutes) }\end{array}$ & Fréquentation & Ville & Localisation \\
O1 & Après-midi & 45 & 18 & Nantes & Machines de l'île \\
O2 & Après-midi & 90 & 5 & Nantes & Parkour-park et spot Fonderies \\
O3 & Après-midi & 90 & 4 & Nantes & Spot Média \\
O4 & Soirée & 100 & 3 & Nantes & Secteur Conservatoire \\
O5 & Après-midi & 95 & 3 & Rennes & Mail François Mitterrand \\
O6 & Après-midi & 70 & 4 & Nantes & Parkour-park \\
O7 & Soirée & 50 & 3 & Nantes & Spot Fonderies \\
O8 & Après-midi & 85 & 21 & Nantes & Secteur Conservatoire \\
O9 & Soirée & 90 & 21 & Nantes & Secteur Conservatoire \\
O10 & Après-midi & 55 & 3 & Nantes & Spot Fonderies \\
\hline
\end{tabular}

Table 2. Profil des enquêtés

\begin{tabular}{ccccc}
\hline Code & Sexe & Activité (en lien avec le parkour) & Ancienneté & Lieu de l'entretien \\
PA1 & Femme & Paysagiste & 7 ans & Lieu de travail \\
PA2 & Homme & Urbaniste & 1 mois & Lieu de travail \\
T1 & Homme & Service des sports de Nantes & Lieu de travail \\
T2 & Homme & Service des sports de Nantes & 8 ans & Lieu de travail \\
T3 & Homme & Service des sports de Rennes & 3 ans & Lieu de travail \\
T4 & Homme & Service des parcs et jardins de Rennes & & Lieu de travail \\
AP1 & Homme & Educateur & 13 ans & Café \\
AP2 & Homme & Dirigeant associatif & 10 ans & Café \\
AP3 & Homme & Educateur & 14 ans & MJC \\
AP4 & Homme & Educateur et autoentrepreneur & 15 ans & Lieu de travail \\
AP5 & Homme & Educateur & 6 ans & Lieu de travail \\
P1a & Homme & Autonome & 6 ans & Spot Média \\
P1b & Homme & Autonome & 5 ans & Spot Média \\
P2a & Homme & Autonome et associatif & 3 ans & Spot Ecluse \\
P2b & Homme & Autonome et associatif & 3 ans & Spot Ecluse \\
P3 & Femmes & Autonome & 5 ans & Université \\
P4 & Femmes & Autonome et associatif & 3 ans & Café \\
P5 & Homme & Associatif & 3 ans & Spot Fonderies \\
E1 & Homme & Elu de Nantes (champ sportif) & 4 ans & Lieu de travail \\
\hline & & & & \\
\hline
\end{tabular}

Dans un premier temps, nous présenterons les intérêts à élargir le champ d'étude de la glisse urbaine vers le parkour pour engager cette actualisation de la recherche en matière d'urbanité. Nous proposons, dans un deuxième temps, des aller-retours entre les études sur le skate et le parkour, y compris avec 
notre travail de terrain à Rennes et à Nantes. L'objectif est de mettre en avant l'existence d'un recoupement des actions des pouvoirs publics entre ces deux pratiques du fait de leur complexité d'appréhension et l'évolution des traitements du fait de la diversification des pratiques. Finalement, les parks seront interrogés en ce qu'ils constituent un outil d'aménagement participant au renouvellement de la citoyenneté et de l'urbanité. Le regard se portera sur une récréativité croissante dans les villes et sur la reconsidération des déviances sociales propres aux outsiders (Becker, 1963), notamment dans la perspective des « déviances positives » (Barthélémy, 2005).

\section{Glisse et parkour : objets d'une réflexion pluridisciplinaire}

\subsection{Transgression sociale, subversion spatiale}

Traceur : "On n'est pas en train de jouer, on fait du sport. » Interlocutrice : "Oui, et bien il y a des terrains de sport pour ça! »

Traceur : "Notre sport, c'est pas comme du foot : c'est toute la ville notre terrain d'entraînement. »

Cet extrait d'une joute verbal dont nous avons été témoin entre trois jeunes traceurs et une riveraine (Observation O5) est révélateur des conflits d'usage et des cohabitations à l'œuvre au sein des espaces publics. La place des pratiquants est interrogée et sujette à débat dans la société et dans la ville. En détournant l'usage du mobilier, cette pratique s'écarte des normes sociales dominantes et est subversive. L'activité et ses pratiquants sont alors mis à distance. Pour illustrer cela, il est intéressant de mobiliser une étude menée sur un groupe de skateurs de Philadelphie par Nemeth :

The skateboarders were considered out of place in the space due to the inherently transgressive and alternative nature of their activity. Officials saw their use of LOVE Park as confrontational because public space is viewed predominantly as adult space; in this context these youths are often seen as "out of order". (2006, p. 309).

Le skate, d'après cet auteur, comme le parkour au regard de nos observations, sont des pratiques de transgression sociale et de subversion spatiale. Elles partagent des caractères subversif et juvénile du fait de leur nature transgressive and alternative et de leur investissement de l'adult space. Traceurs et skateurs sont hors de leur « place » dans la ville, plus précisément out of order et out of place vis-à-vis des normes sociales qui régissent les comportements et définissent l'accès aux espaces.

Cette place alternative dans la ville est revendiquée par les traceurs et s'intègre à des réflexions mobilisant certaines théories de l'espace. Le parkour engage un rapport original à l'espace qui "proposes an alternate way of interacting with and interpreting the urban landscape, one that has the potential to generate new gestures »(Ortuzar, 2009, p. 65). Il est basé sur le développement d'un certain regard - la Pk vision - amenant à percevoir une autre dimension de la ville. A l'image de Bornaz (2013), les traceurs prétendent « redécouvrir la ville » (Entretiens AP3 et AP4). Le parkour devient le prisme de perception et de construction d'une image de l'environnement. Ainsi, il est possible de considérer qu'il prend une forme politique au sens grec de la polis, i.e. la vie de la cité, un «rapport au lieu qui combine esthétique et politique suggérant une aspiration à ré-enchanter le monde » (Gibout, 2016, p. 244). Il interroge le fonctionnalisme urbain au regard de la mobilisation de deux théories de l'espace. D'une part, en dépassant symboliquement, en franchissant matériellement et en résistant aux obstacles de la ville capitaliste, le parkour « lisserait » l'espace urbain (Deleuze et Guattari, 1980). D'autre part, la subversion et le ludisme propre aux traceurs participeraient à la « détente » de l'environnement urbain (Stevens, 2007). Ces éléments, revendiqués par les traceurs ou intégrés à une grille de lecture théorique, confirment l'impact social et spatial du parkour sur la ville et son urbanité.

Plus précisément, le parkour prolonge la dynamique de promotion d'une récréativité croissante des sports de glisse. Au même titre que le skate (Michenaud, 2016), il est vecteur d'une urbanité ludique (Adamkiewicz, 2002) en réintroduisant du jeu dans l'espace urbain. En effet, tous deux sont caractérisés 
par une «imprévisibilité »(Gasnier, 1994): détournements sociaux d'usage, dichotomie entre les utilisations préconçues et les usages effectifs et prégnance des jeunes dans l'espace. Or le ludisme est une des dimensions de la récréativité avec le dépassement physique de soi, la redécouverte d'un paysage ou le plaisir de partager un moment de sociabilité. Le parkour intègre à cette urbanité récréative un rapport différent à l'espace urbain d'une part, plus «fort » (Entretiens P1a et P1b) et plus «corporel » (Entretien P4) et, d'autre part, le développement d'une forme d'aventure (Kidder, 2013). Avec une dimension corporelle renforcée par l'absence d'outil extérieur, le corps du traceur est d'autant plus générateur et producteur d'espace (Lefebvre, 2000 ; Di Méo, 2010). Il est objet et sujet de l'écologie humaine, médium des interactions entre l'individu et l'espace et point d'incorporation des normes sociales et attaches territoriales (Di Méo, 2010). L'appréhension spatiale par le corps est particulièrement identifiable chez les traceurs par leur usage des pieds pour évaluer la distance des sauts (Observation O4). Alors, le renouvellement de l'urbanité à l'œuvre ouvre "une fenêtre d'opportunité pour saisir distinctement et différemment les recompositions politiques et citoyennes à l'œuvre dans l'espace urbain» (Gibout et Lebreton, 2014, p. 81). Recompositions observées auprès de traceurs, pratiquants de glisse urbaine et d'autres sports de rue. A cet effet, citons notamment les travaux de Jean Corneloup $(2017$, p. 7) qui montrent que s'opère une transition récréative dans les villes contemporaines décrite comme "un changement radical de société pour réinventer un avenir plus durable et mieux connecté entre les humains, la nature, les organisations et les univers technologiques ».

\subsection{Dimensions revendicatives ludo-sportive et spatiale}

En matière de politique sportive, le parkour et la glisse urbaine font évoluer les considérations. $\mathrm{Ne}$ s'inscrivant pas dans le modèle de développement sportif traditionnel (Turner, 2017), ils impliquent un renouvellement de l'approche par les pouvoirs publics. Celui-ci passe par le dépassement de la vision des sports de rue comme des « sous-cultures » marginales par les instances sportives (Vieille Marchiset, 2003). Deux orientations sont possibles : évoluer de manière subtile et au cas par cas pour intégrer cette marginalité sportive ou engager une recomposition de l'action par sa diversification (Arpaillange, Darlon et Montane, 2001). Avec « Nantes Terrain de Jeux » (NTJ), la municipalité nantaise a opté pour la seconde option à travers une démarche «diplomatique et conciliante » (Entretien T2) intégrant et accompagnant le développement des pratiques de la «culture underground» (Entretien T1). Cette politique publique, lancée en 2016, vise à en améliorer l'accompagnement (promotion, développement, aménagements, événements, etc.) sans dénaturation. A cet effet, citons l'exemple des skate-parks et parkour-parks. Ces projets d'aménagement sont le plus souvent conçus dans le cadre d'une politique de participation citoyenne, comme à Nantes pour celui du quai Doumergue (Entretiens PA1, AP1 et AP2) qui réunit les deux types d'activité. Ils constituent donc des outils permettant une meilleure appréhension des pratiques par les pouvoirs publics. Par exemple, ils participent à l'amélioration de l'évaluation par un comptage des flux de pratiquants (Turner 2017). De plus, l'aménagement de parks s'inscrit «dans le contexte global du développement d'actions intersectorielles et d'une hybridation des formes et des contenus d'intervention des politiques publiques » (Michenaud, 2016, p. 50).

La glisse et le parkour développent également une revendication spatiale sur les questions de partage et d'accès à l'espace. Les pratiquants engagent une démarche politique explicite et implicite. Leurs activités répondent aux besoins des citadins identifiés par Lefebvre (1968) à travers son « Droit à la ville ». L'ouverture, l'aventure, le jeu, l'imprévu, l'immédiateté, se dépenser et percevoir le monde à travers ses cinq sens permettent de s'extraire d'une ville où la vie serait misérable du fait de ses contraintes et prescriptions importantes. La correspondance du parkour et de la glisse à cette thèse n'est ni toujours exprimée ou même consciente chez les pratiquants, mais a déjà été appliquée à l'étude des cultures sportives et urbaines (Lebreton, 2009). C'est en matière d'accès à l'espace, comme ressource d'aménités sociales et d'opportunités spatiales, que la dimension revendicative des pratiques apparait plus concrètement. Tandis qu'il est complexe dans le cadre législatif français, celui-ci est formalisé par l'« Everyman's Right » dans les sociétés scandinaves. Il s'agit d'un droit d'accès de tous et partout dans 
la mesure où il n'y a ni dérangement, ni dégradation ni trace du passage (Ameel et Tani, 2012). Ainsi, il s'avère que le droit à l'espace est une lutte permanente à laquelle la glisse et le parkour participent tous deux, admettant que ce droit n'est jamais totalement acquis.

L'expérience du skate est intéressante pour analyser cette réalité qui touche également le parkour à travers les tensions liées au partage de l'espace. Les conflits d'usage entre les pratiquants et les autres individus se construisent sur une tension représentationnelle et engagent une lutte pour l'acceptation sociale de l'activité par des négociations spatiales. Le schéma suivant (Figure 4) reprend cette logique en s'inspirant de l'exemple du skate à La Plata en Argentine (Savari, Chaves et Machemehl, 2011). Le principal défi pour les pratiquants n'est pas de s'imposer et «conquérir» un territoire de pratique mais d'asseoir la légitimité de leur activité et forme d'occupation de l'espace. Plusieurs aspects facilitent cette négociation. L'appropriation spatiale du parkour et de la glisse est temporaire et éphémère, ce que Gibout (2013) décrit comme une logique d'usufruit plus que de propriété. Lorsque les pratiques sont dans une démarche déambulatoire, elle est transitoire. Pourtant, quelques traceurs défendent, en vain, leur pratique et préfèrent partir pour préserver leur image (Observation O5) dans un conflit représentationnel dont ils ont conscience : «Les gens ont souvent leur référentiel : ils savent qu'eux ne sont pas capables de le faire donc estiment que c'est dangereux. » (Observation O7).

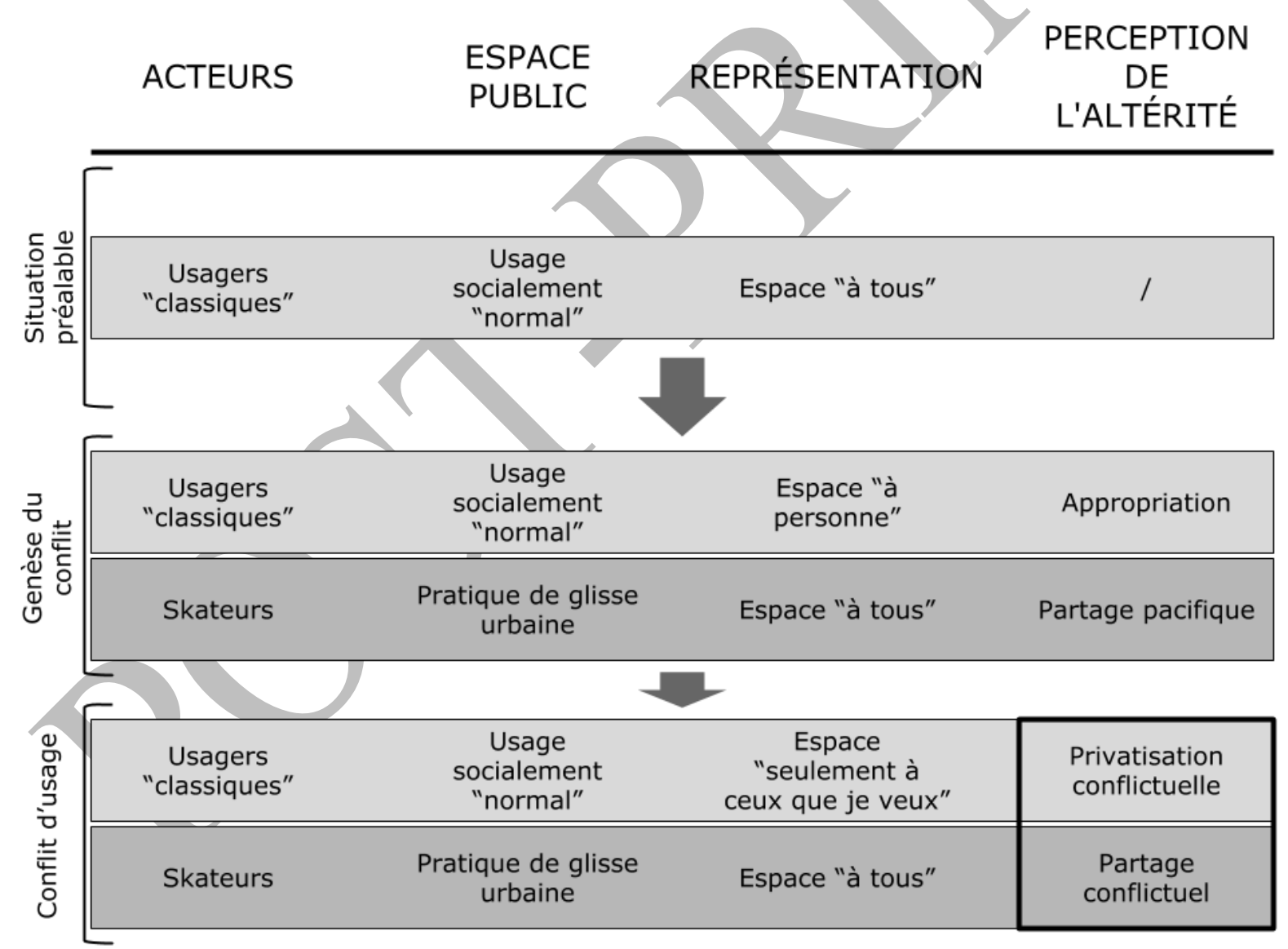

Figure 4. Construction des conflits d'usage de l'espace public

\subsection{Bilan : des pratiques «de $»$ la ville}

Le parkour et les sports de glisse urbaine s'inscrivent dans la lignée d'un questionnement de Di Méo : les interrelations du corps et de l'espace ne constituent-elles pas "les clés de la lecture et de la compréhension de l'espace social, particulièrement de celui des villes? »(2010, p. 487). Les pratiquants 
participent à l'esthétisation de l'environnement en attribuant des significations à l'espace (Ameel et Tani, 2012). L'interaction physique entre l'individu et son milieu engage alors une lecture sensible de ce dernier, par opposition au fonctionnalisme (Lebreton et Andrieu, 2011). Ce processus implique un entraînement intensif (Miaux, 2009) au contact des divers défis que présente la ville. Traceurs et riders répètent leurs figures en park mais aussi sur des lieux qu'ils s'approprient le temps de l'activité, variés pour "diversifier les situations et changer d'environnement»(Observation O1). Pour toutes ces activités, il est possible de mobiliser le concept d'affordance ${ }^{2}$, i.e. le «dépassement » des usages socialement construits et perçus des éléments de l'environnement par la pratique, transformant les obstacles et contraintes en opportunités (Lamb, 2011). C'est à travers le couple perception-action que cette réinterprétation de l'environnement s'opère. Les actions résultent de l'évaluation des possibilités selon les aptitudes corporelles, l'environnement physique et la sphère sociale (Lebreton et Andrieu, 2011). Investissement physique et détournement de l'utilisation des éléments de l'espace sont des composantes essentielles de l'appropriation spatiale à l'œuvre à travers le parkour et la glisse urbaine, plus encore de la production de l'espace puisque «c'est à partir du corps que se perçoit et que se vit l'espace, et qu'il se produit » (Lefebvre, 2000, p. 190). Cet usage de l'espace entraîne l'attribution d'un sense of place (Ameel et Tani, 2012) qui renforce l'attachement et la significativité des lieux (Entretiens AP5 et P3). L'appropriation spatiale décrite est donc autant physique que symbolique, les traceurs évoquant notamment l'importance du "respect de l'espace " dans leur pratique (Entretiens AP2 et AP5). Comme pour le skate, le lieu d'évolution est dans un premier temps produit par les architectes et urbanistes, puis reproduit par ses usagers (Chiu, 2009).

Alors qu'une pratique « dans » la ville s'y déploie, une pratique « de » la ville y devient légitime (Gibout, 2013). Dans le cas du parkour, « our body is not primarily in space: it is of it » (Lamb, 2011, p. 171). Cette classification est motivée par trois principaux arguments : le caractère communautaire, démontré pour la glisse et à confirmer pour le parkour ; le caractère alternatif, voire marginal, par rapport aux usages normatifs de l'espace public - notamment pour le parkour - et au mouvement sportif traditionnel - plus marqué pour les sports de glisse - ; et enfin la dimension revendicative abordée plus avant. La communauté du parkour est concrètement visible à travers la volonté de rassemblement malgré la diversité interne des formes de pratique. Les réseaux sociaux y participent. Le groupe Facebook «Parkour Freerun ADD Nantes » rassemble près de 1000 membres qui y débattent et y organisent leurs sessions. Les valeurs partagées, convergeant sur «la recherche de liberté » (Entretiens AP4 et AP5) et d' "émancipation » (Entretien AP4) et la volonté de " dépasser les barrières de la société » (Entretien AP4). La socialisation des traceurs s'articule donc dans un entre soi de partage d'idées, valeurs, expériences et styles de vie (Lebreton, 2009). Elle crée des espaces de sociabilité appropriés marqués par des dynamiques identitaires individuelles et collectives relevant du concept de «lieu anthropologique » (Augé, 1992), théorisé pour le parkour (Lebreton et Héas, 2010) et pour le skate (Cretin, 2007; Machemehl et Sirost, 2011). Quant au caractère alternatif du parkour, il est mis en avant par les édiles qui ne peuvent encourager, ni même cautionner, "la prise de risque inhérente à certaines formes de pratique » (Entretien T2). Il s'observe par la construction de micro-espaces, assimilables à des hétérotopies (Foucault, 1984), où le temps ne devient plus relatif qu'à la pratique et où les normes sociales qui régissent l'espace urbain sont dépassées. A l'image de la figure 5, nos investigations nous ont menés dans un jardin public après son horaire de fermeture (Observation O1) et sur une aire de jeux pour enfants de 3 à 12 ans (Observation O10). 

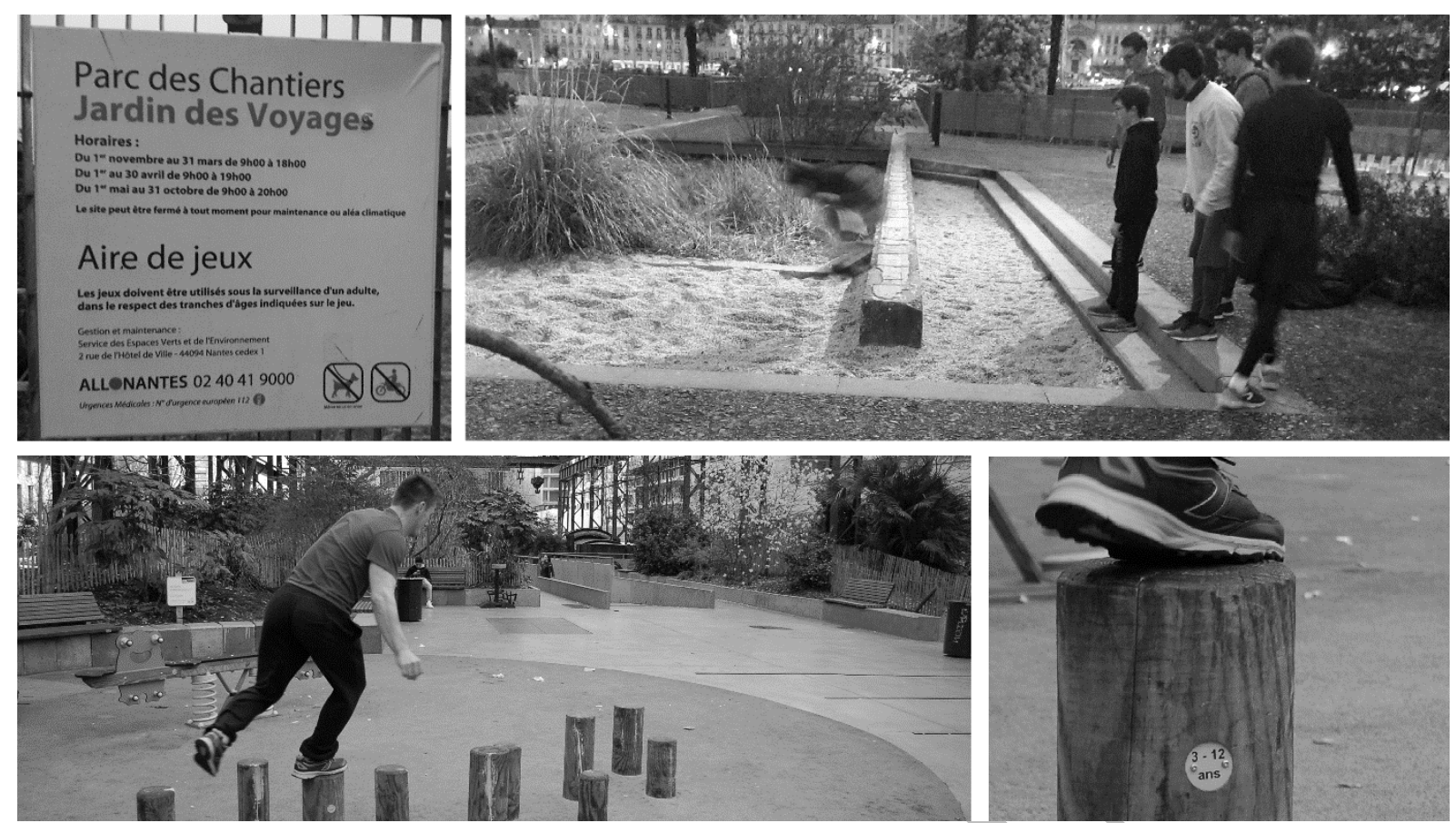

Figure 5. Le parkour, construction d'espace-temps «hors» de la ville sociale et spatiale telle une hétérotopie ? (Robin Lesné, 2018)

\section{Du skate-park au parkour-park, entre reproduction et diversification à Nantes et à Rennes}

Le cadre théorique général étant posé et justifiant l'intérêt de mobiliser l'étude du parkour pour mettre au jour la recherche sur les urbanités sportives, il convient de se focaliser sur l'objet central de notre réflexion. L'intérêt se tourne vers les espaces aménagés et dédiés aux sports de glisse urbaine ou au parkour. Plus précisément, c'est au prisme du rapport à l'institutionnel qu'est analysé cet objet.

\subsection{Une appréhension complexe menant à des traitements institutionnels multiples}

Le parkour, comme la glisse urbaine à ses débuts, est un objet atypique qui met les pouvoirs publics en difficulté. L'appréhension des pratiques, préalablement nécessaire à toute démarche institutionnelle, s'avère complexe. L'objectif est alors de construire une représentation consensuelle entre les services municipaux (Adamkiewicz, 2001). Nous avons identifié trois grilles de lectures mobilisées par les agents des collectivités locales pour y parvenir. La première se base sur l'observation de la dominante juvénile et incite à une démarche d'encadrement. Cette initiative, perceptible à travers les projets de park, semble être en dissonance avec les motivations d'émancipation et d'auto-organisation des jeunes. Ils préfèrent l'appropriation des espaces à l'utilisation de ceux conçus spécifiquement pour eux (Nemeth, 2006) et souhaiteraient «faire remonter les dégradations et autres problèmes et pouvoir proposer des améliorations, évolutions et modifications de modules » (Entretien P2a). La deuxième grille de lecture est celle de la «désorganisation sociale » (Vulbeau et Barreyre, 1994). Elle se traduit par une démarche de « normalisation » : accompagnement du montage associatif, «sportivisation » et définition d'espaces dédiés à la pratique. Enfin, les acteurs municipaux ont tendance à aborder le parkour au prisme des sports de glisse urbaine, activités qu'ils maitrisent davantage. Les traceurs s'attachent pourtant à en garder une certaine distance pour limiter les confusions et traitements inadaptés. En effet,

Parkour is closer to free climbing, a sport in which individuals "flow" by moving their bodies in a delicate relation with their immediate surroundings, without the use of any tools. Much like the climbers, parkouristes must develop a certain sensitivity to gravity's pull and channel its forces to different muscles of the body. (Ortuzar, 2009, p. 57) 
Pour autant, les pouvoirs publics présentent une volonté de reconnaissance institutionnelle. Les professionnels ayant travaillé avec des traceurs mettent en avant plusieurs limites : la nature d' "une pratique urbaine à la base pas forcément faite pour être encadrée » (Entretien PA1) et la volonté de la pratique à "rester indépendante pour ne pas se faire absorber » (Entretien AP4). En matière d'action publique en direction des jeunes, Bier (2007, p. 60) définit la «politique de la reconnaissance » comme "une forme de reconnaissance de la jeunesse, qui se traduit par son entrée dans l'espace public, au sens où l'entend Habermas, et par la participation à une forme de démocratie délibérative ». Les politiques publiques NTJ et «Nantes\&Co », cherchant à faire des pratiquants les acteurs centraux des projets, s'inscrivent dans cette démarche. En matière de politique municipale en direction de la jeunesse, trois types de fonctionnement institutionnel sont identifiés par Bordes (2007) : ouvert, en interaction avec les groupes concernés par des projets et actions ; figé, dénué d'échange et à l'origine de la fuite ou du conflit avec le public en question ; et fermé, désintéressé de ces groupes alors ignorés. Nantes développe une politique ouverte en direction du parkour tandis que Rennes s'inscrit dans un fonctionnement davantage fermé (Lesné, 2018). L'étude sur les pratiques sportives juvéniles dans des quartiers urbains défavorisés (Arpaillange, Darlon et Montane, 2005) a fait émerger l'idée d'une « institutionnalisation différenciée ». Si pour la municipalité rennaise la prise en charge du parkour est contrainte et marginale, la ville de Nantes montre un certain volontarisme pour «faciliter l'entrée dans la pratique » (Entretien T1) et « accompagner le développement de la pratique » (Entretiens T2 et E1).

Table 3. Résumé du traitement institutionnel du parkour par les municipalités

\begin{tabular}{lcc}
\hline Fonctionnement (Bordes, 2007) & Nantes & $\begin{array}{c}\text { Rennes } \\
\text { Institutionnalisation (Arpaillange, Darlon \& Montane, 2005) }\end{array}$ \\
Reconnaissance (Bier, 2007) & $\begin{array}{c}\text { Politique } \\
\text { ouverte } \\
\text { Volontaire }\end{array}$ & Colitique fermée \\
& $\begin{array}{c}\text { Participation } \\
\text { démocratique }\end{array}$ \\
\hline
\end{tabular}

\subsection{L'outil du park, gradient de l'inclusion à l'exclusion sociale}

Les espaces dédiés sont un outil stratégique de traitement des activités pour les services municipaux, entre inclusion, encadrement et exclusion. Il est sujet d'inclusion, et non d'intégration, car les parks alimentent un double processus d'adaptation pour l'entrée des pratiques dans la ville. D'une part, les ludo-sportifs acceptent de changer, en s'adaptant à l'affluence massive d'enfants dans les skate-parks par exemple (Riffaud, Gibout et Recours, 2016). D'autre part, la société urbaine tolère une remise en question de ses normes et habitudes dominantes. Outil d'inclusion, le park participe à la démocratisation des activités. Relevant de la troisième génération d'espaces sportifs ouverts dans la ville (Vieille Marchiset, 2007), il favorise le développement des pratiques par l'offre d'un lieu accessible et socialisant. Il participe à la promotion de l'activité, les néophytes pouvant visualiser la pratique pour se construire des repères stables. Les parents sont rassurés par l'environnement sécuritaire - également cité par certains pratiquants (Entretiens P1a et P2b) - et localisable mis en place (Shannon et Werner, 2008) : "Certains parents craintifs confient que les comportements qu'ils ont observés et ce que les enfants en racontent les ont convaincus que les skateparks sont des espaces sécurisés et qui peuvent même avoir une bonne influence sur leurs enfants » (Riffaud, Gibout et Recours, 2016, p. 34). L'inclusion sociale par les espaces dédiés passe également par la récurrence de la démarche de participation citoyenne des pratiquants locaux dans leur conception, ce qui accroit les relations avec les institutions. À Nantes, le park du quai Doumergue (Figure 1) a été co-conçu par des membres d'associations de parkour Art in Motion et de glisse Unity4Ride et Azymut. Celui du campus universitaire a été réalisé par 4Pk, une entreprise créée par un traceur nantais et spécialisée dans l'aménagement d'espaces dédiés. 
Vecteur d'inclusion sociale, les espaces dédiés peuvent également permettre d'encadrer voire de contrôler les actions des traceurs. Face à la question du risque, le parkour-park peut devenir un outil sécuritaire. Au Royaume-Uni, son développement a été accompagné d'une mesure législative : la volenti non fit injuria (Gilchrist et Osborn, 2017), une clause déchargeant l'usage de l'installation aux risques et périls des usagers. Ainsi, existe une vraie volonté d'encadrer les groupes de pratiquants. Les parks permettraient de maitriser voire restreindre les comportements (Turner, 2017), ou relèveraient du moins d'une stratégie de déplacement de la pratique (Ameel et Tani, 2012). Nous avons pu rencontrer des traceurs qui s'inscrivent dans cette vision, considérant que confiner la pratique dans un park c'est comme "mettre un animal en cage (Entretien P5) car «ça fait un peu zoo, on met les singes dans un parc » (Entretien P3).

Au contraire, les skateparks participent selon nous à l'exclusion du jeu et des activités ludosportives de l'urbain. Il est manifeste, et certains élus ne s'en cachent pas, que la construction d'un skatepark contribue aux multiples opérations de contrôle de l'espace public. Il permet d'éviter les aléas liés aux enfants et à leurs jeux dans une ville qui se veut et se pense comme rationalisée, sécurisée, voire sécuritaire. (Riffaud, Gibout et Recours, 2016, p. 38)

En effet, il est possible de considérer que les parks sont des outils d'exclusion socio-spatiale. Le développement des skate-parks dans les années 1990 répondait à une volonté d'isoler les pratiquants et de les reléguer dans des espaces périphériques. La "tendance générale était plutôt celle d'une fragmentation des usages et d'une segmentation de l'espace public »(Michenaud, 2016, p. 36). En plus de cette relégation spatiale, l'espace dédié entraîne également une exclusion davantage sociale. Vieille Marchiset explique, à partir du cas de Besançon (2003), que les skate-parks participent à une assimilation de la pratique qui la rend «clandestine » hors de l'équipement. Une forme de containment, donc d'enfermement spatial et symbolique de l'activité. Des interprétations similaires sont construites dans les cas du parkour (Gilchrist et Wheaton, 2011) ou du roller à travers la métaphore de la boîte de pétri (Riffaud, 2017). Elles nous invitent à analyser les espaces dédiés au regard d'une géographie de l'exclusion (Sibley, 1995). Celle-ci postule que certains dispositifs construisent ou renforcent la différence d'un objet, l'inscrivant dans une transgression justifiant son exclusion. La quasi-absence de considération du parkour par la politique rennaise «fermée » illustre une réflexion très peu avancée. L'hypothèse du dispositif différenciant n'est donc pas vérifiable. Du côté nantais, la politique « ouverte » NTJ, affichant une volonté de collaboration entre les pratiquants et l'institution, s'inscrit dans une démarche d'affirmation du caractère différent de l'activité. Selon une logique politique assez classique combinant ouverture et fermeture, les parks jouent alors un double rôle de reconnaissance du parkour mais d'exclusion de sa forme hors structure et hors espace dédié.

\subsection{Le park, source de divergences dans les communautés pratiquantes}

Les logiques spatiales du skate et du parkour se concentrent dans des «hauts-lieux ». Ces spots sont "porteurs d'un capital symbolique» (Vieille Marchiset, 2007, p. 150) du fait de la qualité des matériaux, de la diversité morphologique et de la nature des autres usagers (Adamkiewicz, 2001). Les choix de localisation du skate sont motivés par l'accessibilité, la visibilité et les caractéristiques techniques (Chiu, 2009 ; Savari, Chaves et Machemehl, 2011). Les traceurs sont à la recherche d'une stimulation issue de la variété des hauteurs, formes et textures et d'une dimension pratique évaluée par la stabilité, la solidité et l'adhérence du mobilier. Les espaces dédiés sont au cœur d'un débat dans les communautés pratiquantes. Les skate-parks ont été rejetés par le public-cible dès qu'ils se sont avérés inadaptés à l'auto-organisation et éloignés des fonctionnalités attendues (Shearer et Walters, 2015). Dans le monde du parkour, l'opposition aux parks est incarnée par des traceurs militants qui avancent divers arguments. Parmi eux, la dérive sécuritaire (Bornaz, 2013) : le parkour-park donne l'illusion de sécurité, donc d'absence de risque dans une situation dangereuse, alors que le parkour apprend à éliminer le danger en maitrisant le risque. 

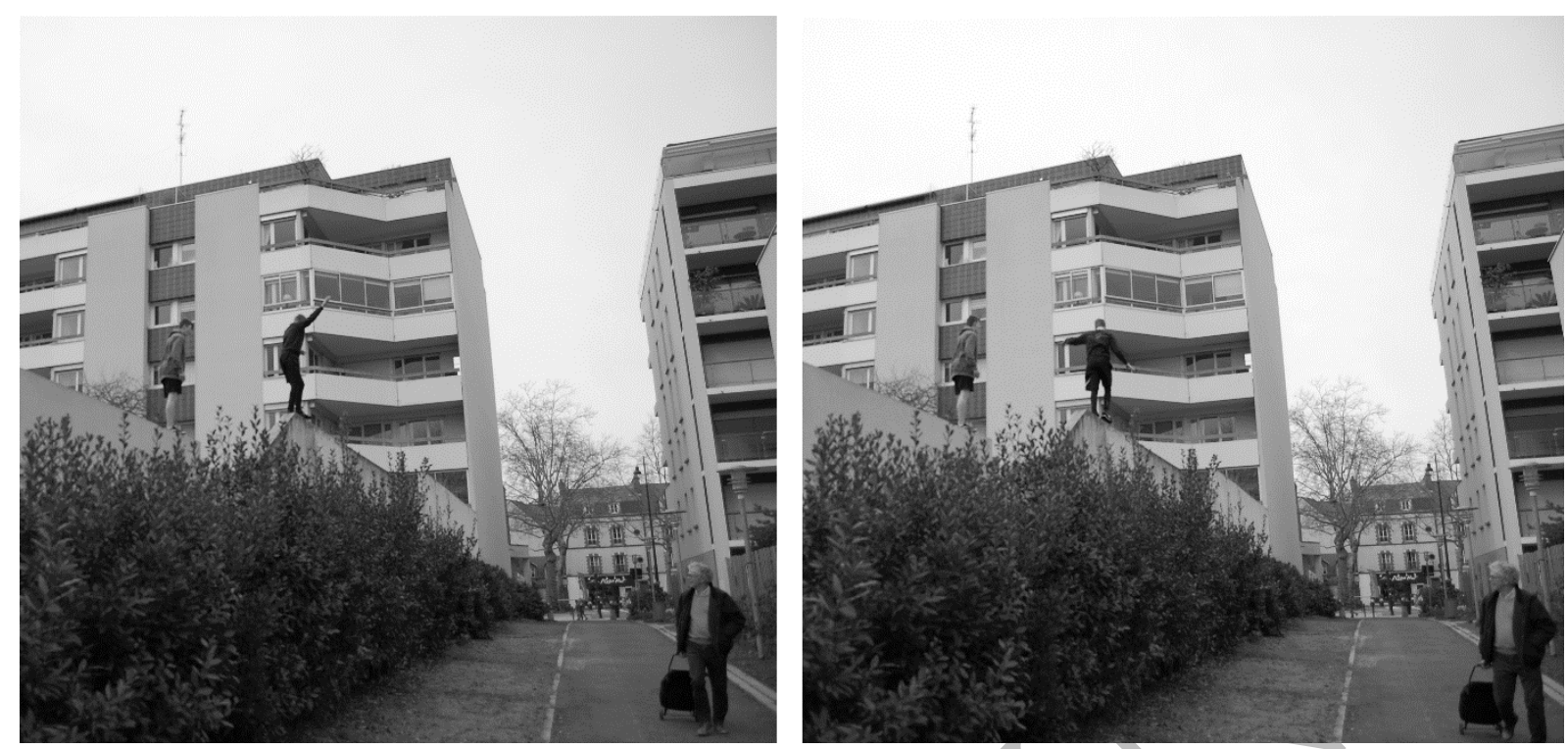

Figure 6. L'équilibriste à 3 mètres de haut, situation dangereuse et maîtrise précise du risque (Robin Lesné, 2018)

L'espace dédié comme projet urbain favorise la participation citoyenne des pratiquants. En participant à sa conception, les jeunes s'approprient en amont l'espace et en développent une acceptation accrue (Entretien T2). Cette assurance d'attachement est vérifiée du côté du skate en Angleterre (Woolley, 2006) et à Marseille (L'Aoustet et Griffet, 2001) et dans le parkour à Jyväskylä en Finlande (Ameel et Tani, 2012) où la réussite est attribuée à l'intégration des pratiquants et de leurs suggestions techniques. Traceurs et riders expriment une volonté de s'investir dans la production - et le suivi (P2a) - des espaces dédiés. Des exemples de skate-parks à Dunkerque (Gibout et Lebreton, 2014) et à Besançon (Vieille Marchiset, 2003), ainsi que l'initiative de l'association nantaise Art in Motion au sein du projet de réaménagement des berges Nord de l'Ile de Nantes (Entretien PA1) illustrent cette volonté. Critique à ce sujet, Bornaz (2013) qualifie l'action publique de cette participation citoyenne des pratiquants de "fatras invraisemblable d'illusions et de mystifications ».

Les parks soulèvent également un enjeu de fond dans les communautés pratiquantes, touchant à la philosophie même de chaque activité. L'espace dédié participe-t-il à l'élargissement et à la diversification de la pratique? Est-il plutôt une «trahison» de celle-ci puisque «toutes les formes de pratique ont peur d'être absorbées et de voir la logique interne de leur discipline modifiée » (Entretien AP4) ? Il s'agit d'une problématique prégnante dans le parkour. La pratique fait art en faisant voir et percevoir le réel différemment, ce qui est en inadéquation avec l'espace dédié qui évincerait cette dimension politico-artistique (Bornaz, 2013). Les traceurs rencontrés évoquent le potentiel limitatif d'un tel aménagement, entre « enfermement » (Entretiens AP2, AP5), confinement physique (Entretiens P2a, $\mathrm{P} 2 \mathrm{~b}, \mathrm{P} 3$ et $\mathrm{P} 5$ ), encadrement législatif (Entretiens AP2, AP4 et $\mathrm{P} 2 \mathrm{~b}$ ) et récupération sportive (Entretiens AP2, AP5, P1a, P1b, P2a, P2b et P3). Cependant, ce débat entraîne davantage d'interrogation qu'il n'est à l'origine d'un militantisme. Les skate-parks ont aussi leurs détracteurs, dénonçant la sur-fréquentation d'un public débutant (L'Aoustet et Griffet, 2001) et sa destination à un public jeune et néo-pratiquant uniquement (Riffaud, Gibout et Recours, 2016 ; Taylor et Khan, 2011 ; Turner, 2017). Un consensus implicite se construit alors dans l'acceptation que le park n'est qu'un spot parmi les autres (Entretiens AP2 et P2b) et le maintien des activités dans leur forme street. Le spot spontanément approprié pour ses qualités ne peut pas être remplacé. Les traceurs modèrent leur critique des parks et admettent qu' " il n'y a pas de pratiquant qui ne fait que du park» (Entretien P1a), ce point de vue se retrouvant également dans le skate (Nemeth, 2006 ; Chiu, 2009) ou d'autres sports de rue (Genelot, 1998). De fait, les parks 
répondent à des besoins spécifiques des pratiquants. L'intérêt est technique pour le skate (Shannon et Werner, 2008) comme pour le parkour puisque «les parks complètent bien la pratique, c'est une alternative à la salle et à tout ce qu'on fait dans la rue » (Entretien P1b). Ils légitiment également la place de l'activité de glisse dans la ville (Turner, 2017) et l'acceptation sociale du parkour : certains traceurs du sud de l'Angleterre "were seen as a nuisance by the (largely elderly) public and police, so they were unable to practice» (Gilchrist et Wheaton, 2011, p. 116) jusqu'à l'aménagement d'un parkour-park.

\section{Rider et tracer en park, activité vectrice d'une citoyenneté juvénile renouvelée}

La thèse avancée par l'intégration du parkour dans la réflexion sur l'urbanité sportive repose notamment sur la dominante juvénile dans les pratiques. Celle-ci, présentée dans certains travaux sur le skate (L'Aoustet et Griffet, 2001 ; Nemeth, 2006 ; Shannon et Werner, 2008 ; Savari, Chaves et Machemehl, 2011 ; Taylor et Khan, 2011), est particulièrement vérifiée dans le cas du parkour (Gibout et Lebreton, 2014), y compris sur nos terrains rennais et nantais (Lesné, 2019). Dans une démarche davantage sociologique et en recentrant le regard sur l'échelle des individus, nous proposons d'interroger (1) le park comme outil de participation citoyenne et (2) la pratique comme levier du renouvellement de l'investissement citoyen des jeunes.

\subsection{Contexte d'émergence de cette citoyenneté : de la déviance à la dissidence récréative}

Les pratiquants de ludo-sports développent un esprit d'autodétermination visible par leur tendance à rechercher une certaine émancipation des institutions et autonomisation des activités et des lieux de pratique (Adamkiewicz, 2002). Cette dynamique se retrouve dans la politique NTJ qui considère que tout projet doit être "à l'initiative des pratiquants qui doivent le porter avant, pendant et après » (Entretien T1). Elle induit un accroissement de la participation et invite à repenser la gouvernance territoriale sur trois points (Lesné, sous presse) : priorisation de l'échelle micro-locale, accroissement de la tolérance par la société et renforcement de l'écoute et de la considération des pratiquants. Ces pratiques entrent donc dans le champ politique et entraînent avec elles les jeunes, catégorie à considérer dans la vie de la cité car s'investissant dans l'espace public et participant à la démocratie dans sa conception habermassienne. Ils constituent en effet des experts locaux incontournables d'une « so-called "street democracy" [...] formed in great part by youth [...] inventing alternative political expression channels as opposed to the official ones »(Lattes, 2004, p. 241). Les pouvoirs publics s'orientent alors vers la proposition d'instruments permettant aux jeunes d'avoir un rôle actif dans la démocratie participative pour «faire des gens des acteurs plus que des consommateurs (Entretien T2) de leurs activités et espaces d'évolution. Ce renouvellement répond à la demande juvénile de démocratie directe. Elle s'inscrit dans une revalorisation de la quotidienneté de la pratique spatiale des jeunes "which focuses on everyday citizenship activities and "ordinary politics" in the lives of young people, particularly as it relates to creative and cultural practices »(Baker, 2015, p. 1000).

Alors, il devient intéressant d'analyser le mode de construction de l'entrée des jeunes dans le champ politique. Pour cela, il est possible de reprendre la considération du skate et du parkour comme des " déviances sociales », à l'image des outsiders (Becker, 1963), pour illustrer le basculement d'une marginalité récréative à une forme nouvelle de citoyenneté. Chaque "pratique est donc déviante si elle s'écarte des normes conventionnelles » (Barthélémy, 2005, p. 168), i.e. en divergence de ce qui est socialement attendu sans nécessaire inscription dans l'illégalité. Cependant,

Déroger à la règle, c'est lutter pour la reconnaissance de sa propre référence. La transgression est ainsi le reflet d'un conflit de légitimité. Sous un autre angle, cela revient à parler de déviance comme une composante positive du fonctionnement organisationnel. (Lebreton, 2015, p. 36). 
Le basculement de considération se situe précisément sur cette acceptation originale de la déviance : la dimension subversive de l'écart à la norme devient constructive et illustre une interaction de l'individu avec son territoire, son identité et son environnement (Lesné, sous presse). "Cette déviance sera positive dans la mesure où elle n'est pas répréhensible ni sanctionnée socialement » (Barthélémy, 2005, p. 168169 ) et fait du parkour "une manière de "transgresser» (de manière positive) les espaces publics urbains et de jouer avec les règles pour mieux les comprendre » (Lebreton, 2015, p. 33). La réflexion reprend une étude menée auparavant sur les dissidences récréatives de nature qui sont caractérisées par "un objectif principal : la critique (éthique, politique et esthétique) ou, dans une moindre mesure, la contestation des espaces de la vie quotidienne » (Bourdeau et Lebreton, 2013, p. 2). Cette perspective s'applique aux pratiques du skate et du parkour de par leur dimension subversive $a$ priori. Elles revêtent bien les caractères politique - par leurs dimensions socio-spatiales hors-normes - et idéologique - à travers leurs cultures sportives et récréatives - propres à la notion. La citoyenneté juvénile en construction relève alors d'une action à finalité récréative sur l'espace public et/ou ouvert de la ville et est caractérisée par le refus de reconnaissance et de soumission à l'autorité politique et/ou idéologique.

\subsection{Construction citoyenne par le projet d'aménagement d'un espace dédié}

L'activité comme dissidence récréative juvénile participe à l'affirmation sociale et spatiale d'une place des jeunes dans la ville. Leur intégration aux projets d'aménagement d'espace dédié est notamment vectrice de participation citoyenne. La municipalité nantaise s'inscrit dans une démarche d'éveil d'une conscience civique avec l'idée que «solliciter les jeunes construit le citoyen » (Entretien AP1) mise en application dans le projet de park du quai Doumergue. Pour cela, elle peut s'inspirer de l'expérience britannique qui démontre que la reconnaissance des lifestyle sports favorise l'engagement et la participation des jeunes, donc leur inclusion sociale (Lebreton, 2016). Cette participation permet d'activer la citoyenneté juvénile, thèse défendue à partir d'une étude sur la parkour dans le sud de l'Angleterre où les "teenagers have been involved with forms of civic engagement " (Gilchrist et Wheaton, 2011, p. 125). Ils ont obtenu une réelle légitimité auprès des pouvoirs publics : «their resourcefulness, maturity, self-direction and creativity positioned them in the eyes of leisure providers and community stake holders as "good citizens"” (Gilchrist et Wheaton, 2011, p. 125). L'investissement citoyen des jeunes dans les projets d'espaces dédiés est une opportunité de revendication et de rénovation démocratique au profit d'un système d'avantage participatif comme mode alternatif d'influence des politiques locales (Bordes, 2007). En effet, le contexte est celui d'une crise contemporaine du système représentatif auprès des jeunes. Une traceuse rencontrée dénonce l'utilisation politique de l'image du dynamisme des jeunes sportifs : «c'est de l'enfumage parce que politiquement c'est pas trop ça : si t'offres pas de futur aux jeunes, ils vont s'énerver et tout faire péter » (Entretien $\mathrm{P} 3)$.

Cependant, cette place des jeunes dans la ville est relativement floue et fluctuante, oscillant entre celle laissée par l'institution et celle que prennent les pratiquants. La politique nantaise NTJ en fait les porteurs de toutes les initiatives, mais "l'enjeu principal c'est l'accès à l'espace public à tous, quelle que soit la pratique »(Entretien E1). Cette politique "dans laquelle chacun peut trouver sa place» (Entretien E1) promeut le «bien-vivre en société » (Entretien E1) et «la liberté dans l'accès à tous » (Entretiens T2 et E1). Elle rejoint en ce sens les résultats d'une étude menée sur le skate à New-York (Chiu, 2009) : la gouvernance de l'espace public doit s'adapter aux changements des besoins des différents usagers et ne pas exclure ceux pour lesquels l'accommodation apparait trop complexe. Le park permet alors l'amélioration des relations entre les pouvoirs publics et les jeunes et l'apaisement des conflits liés au partage de l'espace public, préalablement identifiés dans le cas des skate-parks (Shannon et Werner, 2008). Ainsi, la place laissée par l'institution aux pratiquants dans la ville est, dans sa dimension socio-spatiale, celle de l'espace dédié et, dans sa dimension socio-politique, celle de la participation citoyenne. Or cette place ne correspond que partiellement à celle revendiquée par les ludosports. La citoyenneté se construit à travers l'aménagement du park, i.e. dans la participation des jeunes 
mais aussi par la prise de conscience des limites de ce type de site de pratique. Les espaces dédiés présentent une capacité à limiter l'influence de certaines qualités du parkour dans la construction identitaire - sportive et citoyenne - des jeunes. A cet effet, citons l'autorégulation spatiale - "par exemple, à Média, c'est priorité aux piétons » (Entretien $\mathrm{P} 4$ ) - et comportementale que s'imposent les pratiquants (Ameel et Tani, 2012), la transmission des valeurs comme "être fort pour être utile » (Observations O8 et O9), ou encore l'apprentissage de l'adaptation dans l'activité qui se répercute dans le quotidien puisqu' "en fait, de prendre sa vie en main ... dans le parkour, professionnellement, dans la famille, c'est ça qu'apprend la pratique! (Entretien AP3). Selon cette thèse, la citoyenneté se forge donc par la conception du park avec ses limites et défauts. Illustrée par l'exemple du parkour-park à Nantes, notre thèse mériterait d'être également mise à l'épreuve avec les sports de glisse urbaine.

\subsection{La récréativité au cour de cette citoyenneté renouvelée}

La citoyenneté juvénile semble s'exprimer de manière limitée par l'investissement dans les projets d'aménagement d'espace dédié. Mobilisant l'outil relativement classique de la participation citoyenne institutionnelle, ils ne favorisent pas la pleine expression de la singularité de cette forme citoyenne en construction.

Hors de ces projets, en ce qu'ils sont des dissidences récréatives, les sports de glisse urbaine et le parkour sont vecteurs de relations entre les pratiquants et les pouvoirs publics : de la "participation des jeunes sur les espaces publics urbains naissent alors des interactions, des démarches de concertation entre eux et les acteurs locaux»(Lebreton, 2015, p. 33). Ces interactions (Figure 7) permettent d'aboutir à l'inclusion des pratiques dans les institutions locales et dans la société urbaine. Ce processus ne passe pas nécessairement par la co-conception d'espace dédié, bien qu'elle puisse y contribuer. Le dispositif de concertation du parkour-park du quai Doumergue à Nantes a été pensé pour "intégrer les futurs usagers car ce sera leur espace »(Entretien PA2), visant autant la mise en place de relations pratiquantsinstitution que la reconnaissance sociale par les habitants et édiles de la pratique et de ses espaces. Traceurs et skateurs relèvent majoritairement de l'affiliation sociale alternative (Moreau et Gaultier, 2001) pour cinq raisons. Ils sont fortement marqués pas une socialisation d'entre soi (Fize, 1993), le jeu avec les usages normés de l'espace, la distanciation avec le modèle traditionnel du « club sportif », le jeu avec les limites corporelles (normatives, juridiques) et les caractères éphémère et démonstratif de leur déambulation. Les traceurs revendiquent le non-encadrement de leur pratique par une entité extérieure, à l'image de leur animosité envers la Fédération Internationale de Gymnastique (Entretiens $\mathrm{AP} 1, \mathrm{AP} 2, \mathrm{AP} 3$ et P4). Ils évoquent également une certaine distance avec le monde adulte puisque "Quand t'es jeune, un adulte ne vient pas te parler comme il parlerait à un autre adulte, il te prend de haut et il est agressif. » (Entretien AP1). Les pratiques sont donc bien des dissidences récréatives. Elles participent à l'intégration à la ville et engagent par là un nécessaire renouvellement de l'urbanité. 


\section{PRATIQUANTS PRATIQUE POUVOIRS PUBLICS}



Figure 7. Schéma de la citoyenneté juvénile en action auprès des pouvoirs publics hors des projets d'espace dédié aux pratiques ludo-sportives

La récréativité est le pilier de cette urbanité qui s'impose progressivement, notamment par la glisse urbaine et le parkour, mais aussi à travers d'autres pratiques culturelles urbaines (arts, design, tourisme, etc.). Ces deux activités correspondent à la catégorie « underdoor» des sports de nature décrite comme celle d'activités ayant "en commun de revendiquer des formes dissidentes et transgressives s'appuyant sur une volonté d'autoproduction et d'auto-organisation de leurs lieux et des pratiques » (Mao, Corneloup et Bourdeau, 2013, p. 92) et qui réinvestissent les espaces urbains. Dans cette perspective, elles innovent et la ville "gagne ainsi en légitimité comme territoire de jeu et d'expression récréative underdoor» (Mao, Corneloup et Bourdeau, 2013, p. 93). Il s'engage alors une forme spécifique d'habiter la ville qui construit des espaces vécus, sensibles et symboliques à travers le récréatif (Corneloup, 2014). Les activités impactent la récréativité de l'urbanité car elles ne se limitent pas à être des pratiques sportives. Elles permettent notamment de s'évader tout en étant près de chez soi - dans la dialectique de l'ici/ailleurs (Piolle, 1993) - par la redécouverte et le renouvellement de l'utilisation des espaces du quotidien, du patrimoine local par exemple (Figure 8). En revalorisant les interactions interindividuelles dans l'espace public, en réintroduisant la dimension corporelle sensorielle et kinesthésique dans le rapport à l'environnement urbain et en s'affirmant comme mode de revendication micro-politique, elles opèrent une transition récréative impliquant de "repenser l'espace public via les sociabilités de contact, l'écologie partenariale du corps ou la résistance récréative » (Corneloup, 2017, p. 9). Somme toute, une propension à habiter qualifiée d' « habitabilité récréative » (Corneloup, 2013). Pour toutes ces raisons, le parkour et la glisse urbaine participent à l'urbanité récréative. Cette transition promeut l'aménagement d'espaces dédiés comme les parkour-parks, mais également d'autres espaces «accueillants» pour les activités ludo-sportives dans une logique de ludification de la ville. Ce processus agit aux différentes échelles et aux différents échelons, que ce soit par les pratiques, les 
aménagements, mais aussi, voire surtout, dans les représentations associées chez les acteurs institutionnels.


Figure 8. Redécouverte du patrimoine industriel nantais par le parkour (Robin Lesné, 2018)

\section{Conclusion : le park, outil de citoyenneté ?}

Dans une perspective aménagiste et technique, le park est un espace conçu pour et dédié à certaines activités précises. Il s'agit d'un outil institutionnel sensé rassurer les pouvoirs publics. Les pratiques ludo-sportives émergentes sont confrontées à une appréhension complexe par les pouvoirs publics du fait de leur distance avec le modèle sportif traditionnel. Le park est donc une solution d'aménagement réorientant les activités vers un schéma représentationnel institutionnel maîtrisé (Chiu, 2009). Fort d'une expérience avec les aires de jeu pour enfants et le skate-park, cet outil est autant structuré par les services municipaux qu'il est structurant, permettant l'exercice d'un certain contrôle sur les activités. Quant aux riders et traceurs, ils entretiennent un rapport ambivalent à cet espace dédié. D'une part, certains avantages lui sont reconnus, comme l'offre d'un spot aux caractéristiques techniques intéressantes et le médium d'une démocratisation de la pratique. D'autre part, cet outil est critiqué en ce qu'il devient l'instrument d'une dénaturation de l'activité, l'éloignant de sa philosophie originelle et semant le doute pour son avenir. Dans ce contexte émerge alors une alternative au park. La tendance la plus contemporaine tend à ne plus considérer l'espace dédié comme ce qui fait, seul, référence en matière récréative : l'idée de 《l'espace accueillant» plus polyvalent et intergénérationnel s'impose progressivement. A Rennes comme à Nantes ont été évoqués à ce sujet le concept d' "agora sportive » (Entretiens T2, T3 et T4) et une «volonté de penser des espaces multigénérationnels »(Entretien PA1).

Dans une autre perspective, davantage sociologique et micro-politique, le park constitue un « espace » de citoyenneté. Les différents projets cités s'inscrivent dans une démarche participative volontaire des pouvoirs publics. La conception et parfois la réalisation et l'entretien des parks constituent donc un outil de participation citoyenne. Celle-ci peut-être à l'initiative de la municipalité qui va chercher les pratiquants, ou des pratiquants eux-mêmes qui s'invitent dans les étapes ouvertes comme les réunions publiques. L'exemple de la politique NTJ est original en ce qu'elle se situe à l'entre-deux, formalisant un terrain propice à la participation et un dialogue constant avec les ludo-sportifs sans jamais être à l'origine d'un projet qui vient, finalement, réellement des citoyens. Ainsi, les parks sont, dans les cas étudiés, un outil (1) de l'amélioration des relations entre jeunes et pouvoirs publics et (2) de l'affirmation de la place des jeunes dans la ville. Les ludo-sports étant à dominante juvénile, ce sont ces acteurs qui entrent progressivement, de manière formelle ou non, en dialogue et en échange avec les pouvoirs 
publics et autres institutions, y compris de manière revendicative ou implicite (Lebreton, 2014). Leur place - au moins en partie - se construit ainsi. Les projets de park dans une démarche participative sont, pour eux, la reconnaissance de leur existence et de leur pratique, somme toute le signe (positif) de leur place dans la ville dans ses dimensions spatiale et sociale. Pour autant, une certaine méfiance, voire défiance, persiste. Elle se justifie notamment par la crainte de subir une forme de récupération à des fins politiques. Il s'agirait d'une prise de contrôle de l'espace dédié par les pouvoirs publics qui auraient tendance à n'activer que la forme de citoyenneté qu'ils désirent à travers cet outil, propos vérifiés dans la pratique du skate et du parkour :

In short, the desire to ensure skateboarders are active citizens renders participation in informal spaces illegitimate and therefore improper. In order to be "active citizens", skateboarders must participate in sanctioned (via funding and management) venues and formally organised programmes of activity. Skaters who reject this approach are deemed to be "deviants" and are treated as such, hence their marginalisation by local authorities and the police. (Turner, 2017, p. 20)

Le park constitue donc bien un outil de renouvellement de la citoyenneté, notamment des jeunes car ils sont les plus représentés dans les activités ludo-sportives sujettes correspondantes. Mais, finalement, il n'est que l'outil d'un processus plus large à l'œuvre dans les villes. Une récréativité croissante s'immisce dans l'urbanité, dépassant là son seul caractère sportif. Cette récréativité est alors le levier d'une citoyenneté renouvelée, notamment en ce qu'elle impacte le non-institutionnel et les réseaux parfois non-officiels. Les parks s'intègrent donc à cette évolution, à cette urbanité récréative en développement. Ils en sont un outil aménagiste dont les limites et alternatives se dessinent déjà alors que nous n'en sommes qu'à ses balbutiements.

\section{Notes}

1. Les auteurs remercient les deux évaluateurs rices pour leur travail de conseils et de relecture

2. Le concept d'affordance est l'invention de James J. Gibson (1979). Christophe Gibout (2004) le mobilise pour la première fois en sciences sociales en expliquant que, dans le sport, la capacité des individus à accéder à une structure élaborée d'affordances leur permet d'atteindre une certaine légitimation au sein du corps social pour la préparation à la compétition.

\section{Bibliographie}

Adamkiewicz, E. (2002). Les pratiques récréatives autonomes urbaines et leurs aménagements. Dans O. Bessy et D. Hillairet (dir.), Les espaces sportifs innovants. Tome 1 L'innovation dans les équipements sportifs (p. 153-178). Voiron : Presses universitaires du sport.

Adamkiewicz, E. (2001). Usages récréatifs de la cité et aménagements. Agora débats/jeunesses, 24, $57-$ 62.

Ameel, L. et Tani, S. (2012b). Parkour: Creating Loose Spaces? Geografiska Annaler: Series B, Human Geography, 94(1), 17-30.

Arpaillange, C., Darlon, C. et Montane, M.-A. (2005). La difficile institutionnalisation de la pratique sportive juvénile dans les quartiers urbains. Usages de l'espace public et concurrence administrative. Dans N. Hossard et M. Jarvin (dir.), "C'est ma ville! » De l'appropriation et du détournement de l'espace public (p. 273-284). Paris : L'Harmattan.

Arpaillange, C., Darlon, C. et Montane, M.-A. (2001). Jeunes, sports et espace urbain. Agora débats/jeunesses, 24, 85-93.

Augé, M. (1992). Non-lieux, introduction à une anthropologie de la surmodernité. Paris : Le Seuil. 
Augustin, J.-P. (2002). Les dynamiques socio-spatiales des pratiques sportives. Dans A. Huet et G. Saez (dir.), Le règne des loisirs : loisirs culturels et sportifs, dynamiques sociospatiales (p. 135-164). La Tour d'Aigues : Ed. de l'Aube.

Augustin, J.-P. (2001b). Les jeunes entre équipements et espaces publics. Agora débats/jeunesses, 24, 9-18.

Augustin, J.-P. (1997). Les territoires émergents du sport. Quaderni, 34(1), 129-40.

Baker, A. M. (2015). Constructing citizenship at the margins: the case of young graffiti writers in Melbourne. Journal of Youth Studies, 18(8), 997-1014.

Barthélémy, M. (2005). Les raids-aventures : Une expérience corporelle extrême. Dans O. Sirost (dir.), Le corps extrême dans les société occidentales (p. 163-170). Paris : L'Harmattan

Becker, H. (1963). Outsiders: Studies in the Sociology of Deviance. New York, NY : The Free Press of Glencoe.

Bier, B. (2007). La «politique de la reconnaissance » comme catégorie d'analyse de l'action publique en direction des jeunes. Pensée plurielle, 14, 53-65.

Bordes, V. (2007). Prendre place dans la cité : jeunes et politiques municipales. Paris : L'Harmattan.

Bornaz, N. (2013). Parkour-parks ou parkour : il faut choisir. Repéré/à http://rechercheaction.fr/11 consolable/2013/02/04/parkour-parks-ouparkour-il-faut-choisir/,

Bourdeau, P. et Lebreton, F. (2013). Les dissidences récréatives en nature : entre jeu et transgression. Espaces Temps. Repéré à https://www.espacestemps.net/articles/les-dissidences-recreatives-ennature-entre-jeu-et-transgression/

Certeau (de), M. (1990). L'invention du quotidien 1. Arts de faire. Paris : Gallimard.

Chantelat, P., Fodimbi, M. et Camy, J. (1996). Sports de la cité. Antrhopologie de la jeunesse sportive. Paris : L'Harmattan.

Chiu, C. (2009). Street and Park Skateboarding in New York City Public Space. Space and Culture, $12(1), 25-42$.

Corneloup, J. (2017). Transition récréative et écologie corporelle. Les carnets du LabEx ITEM. Repéré à http://labexitem.hypotheses.org/271

Corneloup, J. (2014). L'habitabilité récréative et écologique des métropoles contemporaines, une ressource territoriale majeure. Dans B. Kadri (dir.), Dynamiques métropolitaines et développement touristique (p. 11-36). Québec : Presses de l’Université du Québec.

Corneloup, J. (2013). Les territorialités sportives transmodernes en émergence. Dans S. Lefebvre, R. Roult et J.-P. Augustin (dir.), Les nouvelles territorialités du sport dans la ville (p. 33-47). Québec : Presses de l'Université du Québec.

Cretin, S. (2007). La transmission des savoirs du skateboard à l'épreuve des nouvelles technologies de l'information et de la communication (Thèse de doctorat, Université de Franche-Comté). Repéré à http://indexation.univ-fcomte.fr/nuxeo/site/esupversions/158107aa-0746-42d6-b626-e2991c8dcffd

Deleuze, G., et Guattari F. (1980). Milles Plateaux : Capitalisme et schizophrénie. Paris : Editions de Minuit.

Di Méo, G. (2010). Subjectivité, socialité, spatialité : le corps, cet impensé de la géographie. Annales de géographie, 675, 466-491.

Fize, M. (1993). Les bandes. L'« entre-soi » adolescent. Paris : Desclée de Brouwer. 
Foucault, M. (1984). Des espaces autres (conférence au Cercle d'études architecturales, 14 mars 1967). Architecture, Mouvement, Continuité, 5, 46-49.

Gasnier, A. (1994). Formes d'urbanité des jeunes et usages des espaces centraux. Travaux et documents de l'URA 915, 1, 35-39.

Genelot, S. (1998). Territoires à vivre : quels aménagements pour les enfants et les jeunes ? Toulouse : Milan.

Gibout, C. (2016). Le parkour... Faire trace dans la ville. Cahiers européens de l'imaginaire, 8, 244247.

Gibout, C. (2013). Territorialités des sports urbains et construction de l'espace public. Dans S. Lefebvre, R. Roult et J.-P. Augustin (dir.), Les nouvelles territorialités du sport dans la ville (p. 67-80). Québec : Presses de l’Université du Québec.

Gibout, C. (2004). De quelques situations délibératives ordinaires dans des pratiques sportives collectives. Dans B. Castagna, S. Gallais, P. Ricaud et J.-P. Roy (dir.), La situation délibérative dans le débat public (vol. 2, p. 177-191). Tours : Presses universitaires François-Rabelais.

Gibout, C. et Lebreton, F. (2014). Cultures juvéniles et loisirs sportifs de rue : une approche par l'espace public. Agora débats/jeunesses, 68, 71-84.

Gibout C. et Mauny, C. (2009). La question locale comme nouvelle frontière socio-spatiale. SociologieS. Repéré à : http://journals.openedition.org/sociologies/2763

Gibson, J. J. (1979). The ecological approach to visual perception. Boston : Houghton-Mifflin

Gilchrist, P. et Osborn, G. (2017). Signposting risk: Parkour parks and the materialities of regulation. Dans D. Turner et S. Carnicelli, Lifestyle sports and public policy (p. 157-179). London : Routledge.

Gilchrist, P. et Wheaton, B. (2011). Lifestyle sport, public policy and youth engagement: examining the emergence of parkour. International Journal of Sport Policy and Politics, 3(1), 109-131.

Kidder, J. L. (2013). Parkour Adventure, Risk, and Safety in the Urban Environment. Qual Sociol, 36, 231-250.

L'Aoustet, O. et Griffet, J. (2001). The Experience of Teenagers at Marseilles' Skate Park. Cities, 18(6), 413-418.

Lamb, M. (2011). Tracing the path of power through the fluidity of freedom: the art of parkour in challenging the relationship of architecture and the body and rethinking the discursive limits of the city (Thèse de doctorat, Bowling Green State University). Repéré à https://etd.ohiolink.edu/!etd.send_file?accession=bgsu1308582200\&disposition=inline

Lattes, G. B. (2004). New forms of public space and new generations. International Review of Sociology, $14(2), 223-243$.

Lebreton F. (2017, juin). Pratiques et usages de la Méthode Naturelle chez les praticiens du parkour : entre filiations et déformations. Communication présentée à la journée d'étude " Demeny-Hébert : La nature en mouvement» de la $2^{\text {e }}$ Semaine Internationale du Corps, Paris, France. doi : 10.13140/RG.2.2.22451.63526

Lebreton F. (2016). Sport de rue et dispositifs de l'action publique. Influxus. Repéré à http://www.influxus.eu/numeros/jeunesse-et-appropriation-de-1/article/sport-de-rue-et-dispositifsde-1-1061

Lebreton, F. (2015). Les sports urbains au service de la médiation éducative. Activités éduco-sportives et cultures urbaines. Les Cahiers Dynamiques, 65, 30-39. 
Lebreton, F., (2014, octobre). Espaces publics et pratiques sportives "alternatives" : vers une démocratie participative ? Communication présentée à la conférence "Sports et cultures sportives en région », Bordeaux, France. Repéré à

https://www.researchgate.net/publication/267920260_Espaces_publics_et_pratiques_sportives_alte rnatives_vers_une_democratie_participative

Lebreton, F. (2009). «Faire lieu » à travers l'urbain: socio-anthropologie des pratiques ludo-sportives et auto-organisées de la ville (Thèse de doctorat, Université européenne de Bretagne). Repéré à https://halshs.archives-ouvertes.fr/tel-00383228

Lebreton, F. et Andrieu, B. (2011). Quand le sport fait corps avec l'espace urbain. Introduction à l'écologie corporelle de la ville. Loisir et Société, 34(1), 99-120.

Lebreton, F. et Héas, S. (2010). Urbanité ludique et cultures sportives : analyse de quatre pratiques physiques de l'espace parisien. Loisir et Société, 33(2), 195-219.

Lefebvre, H., (2000). La production de l'espace (4e éd.). Paris : Anthropos.

Lefebvre, H. (1968). Le droit à la ville suivi de Espace et politique. Paris : Anthropos.

Lesné, R. (2019). Quand le déplacement devient une fin en soi. La pratique du parkour, une mobilité qui fait bouger l'urbanité. Urbanités, 11. Repéré à http://www.revue-urbanites.fr/11-lesne/

Lesné, R. (2018). De la rue au park : le parkour, une pratique juvénile face à l'institutionnalisation. Exemples à Nantes et à Rennes. (Mémoire de Master 2 inédit, Université Rennes 2). doi : 10.13140/RG.2.2.13833.75367

Lesné, R. (sous presse). Le parkour, une pratique au service de la participation juvénile en ville ? Dissidence récréative et affirmation des jeunes à Rennes et à Nántes. L'Information géographique, 83(3).

Machemehl, C. et Sirost, O. (2011). Le sport dans l'espace urbain. Loisir et Société, 34(1), 11-19.

Mao, P., Corneloup J. et Bourdeau P. (2013). L'indoor, l'underdoor et l'aroundoor : les sports de nature envahissent la ville. Dans S. Lefebvre, R. Roult et J.-P. Augustin (dir.), Les nouvelles territorialités du sport dans la ville (p. 81-98). Québec: Presses de l'Université du Québec.

Miaux, S. (2009). Le libre mouvement des corps. Géographie et cultures, 70, 99-116.

Michenaud, H. (2016). Entre autonomie de la pratique et institutionnalisation : le skateboard comme double enjeu spatial et politique. Le cas de Rennes (Mémoire de $4^{\text {ème }}$ année inédit). SciencesPo Rennes.

Moreau, C. et Gaultier, G. (2001). Les jeunes dans l'espace public distants des institutions ? Agora débats/jeunesses, 24, 31-40.

Nemeth, J. (2006). Conflict, Exclusion, Relocation: Skateboarding and Public Space. Journal of Urban Design, 11(3), 297-318.

Ortuzar, J. (2009). Parkour or l'art du déplacement. The Drama Review, 53(3), 54-66.

Piolle, X. (1993). La montagne «ailleurs" privilégié des citadins favorisés. Aménagements et environnements montagnards, 11, 107-111.

Riffaud, T. (2017). Stérilisation ou boîte de pétri ? La politique publique des espaces dédiés aux pratiques du graffiti et de la roule. Dans C. Gibout (dir.), Activités sportives, récréatives et ludiques \& développement des territoires (p. 281-296). Saint-Denis : Edilivre.

Riffaud, T., Gibout C. et Recours, R. (2016). Skateparks : de nouveaux parcs de jeu pour enfants. Les Annales de la recherche urbaine, 111, 30-41. 
Savari, J., Chaves, M. et Machemehl C. (2011). Logiques d'appropriation et politiques de l'espace urbain : jeunes skateurs dans la ville de La Plata en Argentine. Loisir et Société, 34(1), 121-148.

Shannon, C. S. et Werner, T. L. (2008). The Opening of a Municipal Skate Park Exploring the Influence on Youth Skateboarders' Experiences. Journal of Park and Recreation Administration, 26(3), 39-58.

Shearer, S. et Walters P. (2015). Young people's lived experience of the "street" in North Lakes master planned estate. Children's Geographies, 13(5), 604-617.

Stevens, Q. (2007). The Ludic City: Exploring the Potential of Urban Spaces. Londres : Routledge.

Sibley, D. (1995). Geographies of exclusion: Society and difference in the west. Londres : Routledge.

Taylor, M. F. et Khan, U. (2011). Skate-Park Builds, Teenaphobia and the Adolescent Need for HangOut Spaces: The Social Utility and Functionality of Urban Skate Parks. Journal of Urban Design, 16(4), 489-510.

Travert, M. (1999). L'envers du stade le football de pied d'immeuble, une pratique singulière au cœur d'une cité populaire (Thèse de doctorat inédite, Université Aix-Marseille 2).

Turner, D. (2017). Performing citizenship: Skateboarding and the formalisation of informal spaces. Dans D. Turner et S. Carnicelli (dir.), Lifestyle sports and public policy (p. 13-26). Londres : Routledge.

Vieille Marchiset, G. (2007). La construction sociale des espaces sportifs ouverts dans la ville. L'Homme et la société, 165-166, 141-59.

Vieille Marchiset, G. (2003). Sports de rue et pouvoirs sportifs : conflits et changement dans l'espace local. Besançon : Presses Universitaires Franc-Comtoises.

Vulbeau, A. et Barreyre, J-Y. (1994). La jeunesse et la rue. Paris : Desclée De Brouwer.

Woolley, H. (2006). Freedom of the city: Contemporary issues and policy influences on children and young people's use of public open space in England.Children's Geographies, 4, 45-59.

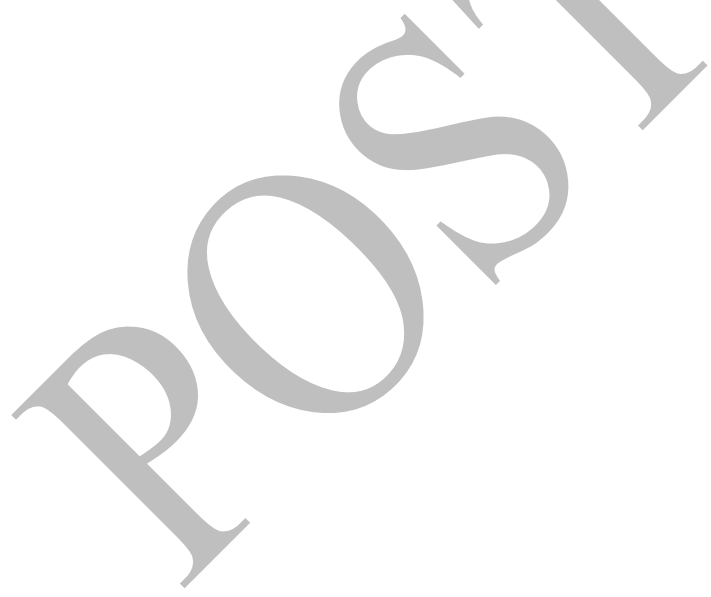

